\title{
Decoherence and discrete symmetries in deformed relativistic kinematics
}

\author{
Michele Arzano ${ }^{1, \star}$ \\ ${ }^{1}$ Dipartimento di Fisica and INFN, \\ "Sapienza" University of Rome, \\ P.le A. Moro 2, 00185 Roma, EU
}

\begin{abstract}
Models of deformed Poincaré symmetries based on group valued momenta have long been studied as effective modifications of relativistic kinematics possibly capturing quantum gravity effects. In this contribution we show how they naturally lead to a generalized quantum time evolution of the type proposed to model fundamental decoherence for quantum systems in the presence of an evaporating black hole. The same structures which determine such generalized evolution also lead to a modification of the action of discrete symmetries and of the CPT operator. These features can in principle be used to put phenomenological constraints on models of deformed relativistic symmetries using precision measurements of neutral kaons.
\end{abstract}

\section{Introduction}

Ordinary time evolution in a closed quantum system preserves purity: given a time evolution operator $S$ the density matrices of the system before, $\rho_{\text {in }}$, and after, $\rho_{\text {fin }}$, the evolution are related by $\rho_{\text {fin }}=$ $S \rho_{i n} S^{\dagger}$. The operator $S$ is unitary, $S S^{\dagger}=1$, and, if the initial state is pure i.e. $\operatorname{Tr} \rho_{\text {in }}^{2}=1$, then $\operatorname{Tr} \rho_{\text {fin }}^{2}=1$ and thus purity is eternal.

The discovery of black hole quantum radiance [1] raised a conceptual puzzle concerning the fate of quantum states after the complete evaporation of the black hole. As Hawking first suggested [2, 3], the full disappearance of a black hole via quantum evaporation, could allow for the evolution of a pure quantum state into a mixed one in contrast with the well established picture recalled above. Hawking's first attitude was to accept de facto this possibility and postulate that in the presence of quantum gravitational effects the unitary evolution operator $S$ should be replaced by a "superscattering" operator $\$$ evolving states according to $\rho_{\text {fin }}=\$ \rho_{\text {in }} \neq S \rho_{i n} S^{\dagger}$ so that starting from a pure state $\operatorname{Tr} \rho_{\text {in }}^{2}=1$ one could in principle evolve to a mixed state $\operatorname{Tr} \rho_{\text {fin }}^{2} \leq 1$

Possible phenomenological signatures of such generalized quantum evolution in neutral kaon systems and for neutron interferometry were first discussed by Ellis, Hagelin, Nanopoulos and Srednicki in [4]. Their approach relied on the analysis of the dynamics associated to $\$$ in terms of a differential equation for $\rho$

$$
\dot{\rho}=\not h \rho \neq-i[H, \rho],
$$

generalizing the usual Von Neumann evolution equation.

^e-mail: michele.arzano@roma1.infn.it 
Building up on the results of [4] Banks, Peskin and Susskind [5] looked for a general form of the evolution term $\not A \rho$ assuming that hermiticity and trace of the density matrix, $\rho=\rho^{\dagger}$ and $\operatorname{Tr} \rho=1$, are preserved by the evolution. The resulting evolution equation they found

$$
\dot{\rho}=-i[H, \rho]-\frac{1}{2} h_{\alpha \beta}\left(Q^{\alpha} Q^{\beta} \rho+\rho Q^{\beta} Q^{\alpha}-2 Q^{\alpha} \rho Q^{\beta}\right),
$$

had been already discovered few years before in the literature on open quantum systems and was known as Lindblad equation [6]. In (2) $h_{\alpha \beta}$ is a hermitian matrix of constants and $Q^{\alpha}$ form a basis of hermitian matrices. In the present contribution we will show how models of deformed kinematics based on Lie group-valued momenta naturally allow for deformed evolution equations of Lindblad type and are thus amenable to phenomenological constraints from precision measurements of fundamental decoherence. Since the seminal work [4] the neutral kaon system has been considered as one of the most efficient candidates to test fundamental decoherence (see e.g. [7] and [8] for a recent review). Over the years it has become clear that these systems provide also a natural testbed for departures from CPT invariance (see Prof. Bernabeu's contribution to these proceedings) and, in this light, in the final part of this contribution we will discuss recent results concerning the formulation of deformed discrete symmetries.

The theoretical framework in which group-valued momenta emerge most naturally is in the study of the kinematics of point particles coupled to gravity in $2+1$-dimensional space-time. In the next Section we will begin with a brief description how how deformed kinematics arises in such setting.

\section{Group valued momenta from three-dimensional gravity}

A well known feature of general relativity in three space-time dimensions is that it does not admit local degrees of freedom $[9,10]$. The geometry of a solution of the theory representing a point particle at rest is described by the conical metric

$$
d s^{2}=-d t^{2}+d r^{2}+(1-4 G m)^{2} r^{2} d \varphi^{2}
$$

where $G$ is the three dimensional Newton's constant (of dimension of inverse mass) and $m$ is the mass of the particle [10].

Such conical geometry is characterized by a deficit angle $\alpha=8 \pi G m$ proportional to the particle's mass and the tip of the cone, a singular point of the geometry, is identified with the location of the particle. In other words due to the topological nature of the theory a localized point particle is represented by a conical space-time defect. This suggests that the kinematical properties of the particle must be encoded in inherently "non-local" quantities capturing their topological nature. Indeed as shown above the mass of the particle $m$ is proportional to the deficit angle $\alpha$ which can be "measured" by calculating the holonomy of the flat connection along a circular path around the defect. Such holonomy is a group element belonging to $S L(2, \mathbb{R})$, the gauge group of the theory, and it is indeed a rotation by $\alpha$ which we denote by $h_{\alpha}$. The rest energy of the particle is thus represented by a $S L(2, \mathbb{R})$ group element (see [11] and [12] for more technical details). According to this picture one would intuitively expect that the general three-momentum of a moving particle will be given by another $S L(2, \mathbb{R})$ group element obtained by "boosting" the rest momentum $h_{\alpha}$. This is indeed the case and it can be shown $[12,13]$ that the three-momentum of a point particle of mass $m$ is given by the group element obtained by boosting with a generic $g \in S L(2, \mathbb{R})$, via group conjugation, the rest three-momentum $h_{\alpha}$ :

$$
h=g h_{\alpha} g^{-1} .
$$


In order to find the actual components of such momentum one needs to introduce a parametrization of the group $S L(2, \mathbb{R})$. A typical choice is to write $h$ using a basis of real two by two matrices given by the identity $\mathbb{1}$ and three linearly independent traceless matrices $\gamma_{\mu}$ :

$$
h=u \mathbb{1}+\frac{p^{\mu}}{\kappa} \gamma_{\mu}
$$

where $\kappa=(4 \pi G)^{-1}$ is a constant with dimension of energy obtained from Newton's constant. The unit determinant condition on $h$ leads to the condition $u^{2}+p^{2} / \kappa^{2}=1$ where $p^{2}$ is the square of the three vector $p^{\mu}$ according to the usual Minkwoski metric with signature $(+,-,-)$. This is nothing but the equation defining three-dimensional anti-de Sitter space, the manifold of $S L(2, \mathbb{R})$, via embedding in a four-dimensional Minkowski space.

The non-abelian Lie group structure of momentum space has dramatic consequences at the quantum level for what concerns the action of symmetry generators on the Hilbert space of the theory. In order to appreciate this let us first recall some basic concepts in elementary quantum theory.

\section{Hopf algebras and quantum mechanics}

In the usual formulation of relativistic quantum mechanics one-particle states belong to a Hilbert space $\mathcal{H}$ which carries a unitary irreducible representation of the Poincare group. Accordingly there is a well defined action of the symmetry generators belonging to the Poincaré algebra on these states. As it is well known, we use such action to label the states, for example in terms of their eigenvalue with respect to translation generators

$$
P_{\mu}|k\rangle=k_{\mu}|k\rangle
$$

where $k_{\mu}$ are on-shell components of the energy-momentum vector. The notion of dual representation of a Lie algebra allows us to define an action of the elements of the Poincaré algebra and in particular of $P_{\mu}$ on the dual Hilbert space $\mathcal{H}^{*}$ spanned by bras $\langle k|$ as

$$
P_{\mu}\langle k| \equiv-k_{\mu}\langle k|
$$

notice that $P_{\mu}\langle k|=\langle k|\left(-k_{\mu}\right)=\langle k|\left(-P_{\mu}\right)$ thus we can define an operation on $P_{\mu}$ called the antipode such that

$$
P_{\mu}\langle k| \equiv\langle k| S\left(P_{\mu}\right)
$$

and thus $S\left(P_{\mu}\right)=-P_{\mu}$.

Analogously one normally defines the action of the symmetry generators on multi-particle states, elements of tensor products of $\mathcal{H}$ in terms of a Leibniz rule. For example for a two-particle state $\left|k_{1}\right\rangle \otimes\left|k_{2}\right\rangle \in \mathcal{H} \otimes \mathcal{H}$ we have

$$
P_{\mu}\left(\left|k_{1}\right\rangle \otimes\left|k_{2}\right\rangle\right)=P_{\mu}\left|k_{1}\right\rangle \otimes\left|k_{2}\right\rangle+\left|k_{1}\right\rangle \otimes P_{\mu}\left|k_{2}\right\rangle
$$

and again we can define an operation on $P_{\mu}$, known as the co-product, which reproduces such action $\Delta P_{\mu}\left|k_{1}\right\rangle \otimes\left|k_{2}\right\rangle \equiv P_{\mu}\left(\left|k_{1}\right\rangle \otimes\left|k_{2}\right\rangle\right)$ and thus

$$
\Delta P_{\mu}=P_{\mu} \otimes \mathbb{1}+\mathbb{1} \otimes P_{\mu}
$$

The operations of antipode and co-product are borrowed from the theory of Hopf algebras but, as we just showed, they are "built in" in our everyday quantum mechanics. 
Two observations are crucial for our purposes at this point. The first is that these basic notions suffice to derive the action of symmetry generators, and in particular of $P_{\mu}$, on a generic operator/observable of the theory. Let us take for example the projection operator $\pi_{k}=|k\rangle\langle k|$ on the state $|k\rangle$. This is a simple yet fundamental operator since it can be employed to express general operator in terms of their one-particle matrix elements [14]. Using the rules recalled above we have

$$
P_{\mu}\left(\pi_{k}\right)=P_{\mu}(|k\rangle\langle k|)=P_{\mu}(|k\rangle)\langle k|+| k\rangle P_{\mu}(\langle k|)=P_{\mu}|k\rangle\langle k|-| k\rangle\langle k| P_{\mu}=\left[P_{\mu}, \pi_{k}\right],
$$

in other words the usual co-product and antipode for $P_{\mu}$ lead to the familiar adjoint action of symmetry generators on operators. The second observation is that the framework of Hopf algebras allows for non-trivial modifications of antipode and co-product which will lead to a deformed adjoint action. Models of relativistic kinematics in which momenta belong to a non-abelian Lie group provide a prototypical example of a modified action of translation generators on states and operators.

Indeed let us consider one-particle states labelled by elements of a non-abelian Lie group $|\pi\rangle$. One can define translation generators $P_{\mu}$ whose eigenvalues on these sates are coordinates functions $\mathcal{P}_{\mu}(\pi)$ on the group manifold

$$
P_{\mu}|\pi\rangle=\mathcal{P}_{\mu}(\pi)|\pi\rangle .
$$

We see that already at the level of dual representation or bra states, the non-trivial group operations enter the game. Indeed one must have that

$$
P_{\mu}\langle\pi|=\mathcal{P}_{\mu}\left(\pi^{-1}\right)\langle\pi| \equiv\langle\pi| S\left(P_{\mu}\right)
$$

in other words the action on bra states (encoded in the antipode $S\left(P_{\mu}\right)$ ) leads to eigenvalues which read the coordinates of the inverse group element $\mathcal{P}_{\mu}\left(\pi^{-1}\right)$. Similarly the action on multiparticle states is non-trivial

$$
P_{\mu}\left(\left|\pi_{1}\right\rangle \otimes\left|\pi_{2}\right\rangle\right)=\mathcal{P}_{\mu}\left(\pi_{1} \cdot \pi_{2}\right)\left|\pi_{1}\right\rangle \otimes\left|\pi_{2}\right\rangle \equiv \Delta P_{\mu}\left|\pi_{1}\right\rangle \otimes\left|\pi_{2}\right\rangle
$$

reflecting a non-abelian addition rule $\oplus$ for the momentum eigenvalues defined as follows

$$
\mathcal{P}_{\mu}\left(\pi_{1} \cdot \pi_{2}\right) \equiv \mathcal{P}_{\mu}\left(\pi_{1}\right) \oplus \mathcal{P}_{\mu}\left(\pi_{2}\right) \neq \mathcal{P}_{\mu}\left(\pi_{2} \cdot \pi_{1}\right),
$$

where $\cdot$ denotes the (non-abelian) group composition. Notice that the non-trivial inverse operation (13) dictated by the antipode is required in order for the following consistency relation to hold

$$
\mathcal{P}_{\mu}(\pi) \oplus \mathcal{P}_{\mu}\left(\pi^{-1}\right)=\mathcal{P}_{\mu}\left(\pi \cdot \pi^{-1}\right)=\mathcal{P}_{\mu}(\mathbb{1})=0
$$

Thus for a Lie group momentum space we have momentum eigenstates of the corresponding quantum system which combine in a non-trivial way reflecting the non-abelian structure of the group. These new rules reflect a deformed action of translation generators on the Hilbert space which are natural in the theory of Hopf algebra generalizations of Lie algebras and Lie groups (see e.g. [15] and references therein).

\section{Deformed translations and Lindblad evolution in three dimensions}

A concrete example of such non-trivial structures is given by the action of translation generators in the case when momentum space is the $S L(2, \mathbb{R})$ Lie group [16]. One can define translation generators [17] whose eigenvalues are given by embedding coordinates $p^{\mu}$ introduced above. In this case the coproduct and antipode read

$$
\Delta P_{\mu}=P_{\mu} \otimes \mathbb{1}+\mathbb{1} \otimes P_{\mu}+\frac{1}{\kappa} \epsilon_{\mu v \sigma} P^{v} \otimes P^{\sigma}+O\left(\frac{1}{\kappa^{2}}\right), \quad S\left(P_{\mu}\right)=-P_{\mu},
$$


where for simplicity we gave only the leading order in $1 / \kappa$ expression for the coproduct while, for this choice of translation generators, the antipode is trivial at all orders in $1 / \kappa$. As we illustrated in the previous section coproduct and antipode determine the action of symmetry generators on generic operators. For symmetry generators belonging to a non-trivial Hopf algebra, as for the case under study, the usual adjoint action on an operator $\rho$ generalizes to the "quantum" adjoint action [18]

$$
\operatorname{ad}_{P_{\mu}}(\rho)=(\operatorname{id} \otimes S) \Delta\left(P_{\mu}\right) \diamond \rho
$$

where $(a \otimes b) \diamond c \equiv a c b$ and id is the identity operator. It is immediate to check that such action reduces to the ordinary commutator for trivial coproduct and antipode. This general expression can be read off the action (11) in the case when the operator $\rho$ is the density matrix describing a pure state and easily generalizes to a generic quantum state.

Let us now focus on the action of the time translation generator $P_{0}$ in order to derive the infinitesimal time evolution equation for the density matrix. Plugging (17) into (18) we obtain

$$
\operatorname{ad}_{P_{0}}(\rho)=\left[P_{0}, \rho\right]-\frac{1}{\kappa} \epsilon_{0 i j} P^{i} \rho P^{j},
$$

leading to a three-dimensional Lindlblad equation

$$
\dot{\rho}=-i\left[P_{0}, \rho\right]-\frac{1}{2} h_{i j}\left(P^{i} P^{j} \rho+\rho P^{j} P^{i}-2 P^{j} \rho P^{i}\right)
$$

where the "decoherence" matrix $h$ is given by

$$
h=\frac{i}{\kappa}\left(\begin{array}{ccc}
0 & 0 & 0 \\
0 & 0 & 1 \\
0 & -1 & 0
\end{array}\right) .
$$

This simple three-dimensional example shows that the non-trivial action of translation generators imposed by the non-abelian nature of the group-valued momenta can naturally accommodate departures from the Von Neumann evolution equation of ordinary quantum mechanics. It is worth noticing that, starting from the seminal work [5], quantum evolution equations of the Lindblad type have always been discussed as the most general linear evolution equations that can allow for the evolution of pure states into mixed states. A main conceptual issue raised by these approaches was the lack of a more fundamental model form which these types of equations could be derived in a low energy limit [19]. Here we showed that, rather suggestively, a Lindblad equation can be obtained from leading order (remember that the $1 / \kappa$ parameter is proportional to the inverse Planck mass) Hopf algebra deformations of relativistic symmetries emerging in three-dimensional gravity.

Below we illustrate how these structures can be generalized to the case of four space-time dimensions.

\section{Deformed symmetries and quantum evolution in four dimensions}

Historically Hopf algebra deformations of relativistic symmetries where first derived for the four dimensional Poincaré algebra. In [20], through a rather involved application of the procedure of quantum deformations of Lie algebras the authors obtained what is now known as the $\kappa$-Poincare algebra. As for the three dimensional case discussed in the previous sections the deformation parameter $\kappa$ carries dimension of energy and can be thought as a UV scale, possibly Planckian if one argues that such deformations emerge from the flat space limit of quantum gravity [21, 22]. It was only 
later realized that these deformations of relativistic symmetries can be interpreted in terms of group valued momenta very much in the spirit of the three-dimensional example discussed above. As was first suggested in [23] and later thoroughly explored in [24, 25] the full structure of the $\kappa$-Poincaré algebra can be obtained in terms of the properties of a momentum space described by the abelian nilpotent group $A N(3)$. Such group can be obtained from the Iwasawa decomposition of the fivedimensional Lorentz group $S O(4,1) \simeq S O(3,1) A N(3)$ [24] and this very structure ensures an action of the four-dimensional Lorentz group $S O(3,1)$ on the group valued momenta. Geometrically the $A N(3)$ group spans a submanifold of the four-dimensional de Sitter space $d S_{4}$ defined in an embedding five-dimensional Minkowski space as the subspace

$$
-p_{0}^{2}+p_{1}^{2}+p_{2}^{2}+p_{3}^{2}+p_{4}^{2}=\kappa^{2} ; \quad p_{0}+p_{4}>0 .
$$

Notice how the deformation parameter $\kappa$ determines the curvature of the momentum manifold.

In full analogy with the three dimensional case one associates translation generators ${ }^{1} P_{\mu}$ to the embedding coordinates $p_{\mu}$. Their co-products and antipodes at leading order in $\kappa$ are given by

$$
\begin{aligned}
& \Delta\left(P_{0}\right)=P_{0} \otimes \mathbb{1}+\mathbb{1} \otimes P_{0}+\frac{1}{\kappa} P_{m} \otimes P_{m}, \\
& \Delta\left(P_{i}\right)=P_{i} \otimes \mathbb{1}+\mathbb{1} \otimes P_{i}+\frac{1}{\kappa} P_{i} \otimes P_{0}, \\
& S\left(P_{0}\right)=-P_{0}+\frac{1}{\kappa} \vec{P}^{2}, \\
& S\left(P_{i}\right)=-P_{i}+\frac{1}{\kappa} P_{i} P_{0} .
\end{aligned}
$$

This choice of translation generators is known as the "classical basis" of the $\kappa$-Poincare algebra [29] since the action of the Lorentz generators on $P_{\mu}$ and the corresponding coordinates $p_{\mu}$ is undeformed and the mass Casimir of the algebra is the usual one $P_{0}^{2}-\vec{P}^{2}=$ const. In other words at the algebra level the structures remain the same as in the ordinary Poincaré algebra and all the non-trivial structures are found at the "co-algebra" level i.e. in the form of the co-products and antipodes. These however will suffice to alter the action of translation generators on operators and, as in the previous section, on the density matrix $\rho$. Indeed a straightforward calculation shows that the time evolution equation is now deformed to a non-symmetric Lindblad equation [16]

$$
\dot{\rho}=-i\left[P_{0}, \rho\right]+\frac{i}{\kappa} P_{m} \rho P_{m}-\frac{i}{\kappa} \rho \vec{P}^{2}
$$

where the $\vec{P}^{2} \rho$ term is missing. Such apparently odd form of the evolution equation is required by the non-trivial form of the antipode for $P_{0}$ (unlike the three dimensional example where it was undeformed). Indeed the antipode enters the expression of the hermitian adjoint of the adjoint action of $P_{0}$ via

$$
\left(\operatorname{ad}_{P_{0}}(\cdot)\right)^{\dagger} \equiv \operatorname{ad}_{S\left(P_{0}\right)}(\cdot)
$$

and one can easily verify that, once this property is taken into account, (23) indeed preserve hermiticty of the density matrix $\rho$ as it must be for a proper quantum evolution equation.

We have thus showed that it is possible to realize a generalized quantum evolution in four spacetime dimensions when translation generators are deformed according to a particular realization of the $\kappa$-Poincaré algebra. In [19] it was claimed that a viable Lindblad-like evolution equation (an equation

\footnotetext{
${ }^{1}$ Choosing different parametrizations of $A N(3)$ leads, in general, to different choices of translation generators corresponding to dfferent "bases" of the $\kappa$-Poincaré algebra (See e.g. [26, 27] and [28] for a possible physical interpretation.)
} 
in which the operators appearing in the decoherence term commute with $P_{0}$ ) would necessary conflict with Lorentz covariance. Is our evolution equation (23) covariant under the action of Lorentz boosts? It turns out that also in this case the role of a non-trivial antipode is fundamental. Indeed it can be shown that, as for $P_{0}$, the adjoint action of boosts on an operator is deformed according to

$$
\operatorname{ad}_{N_{i}}(\rho)=\left[N_{i}, \rho\right]+\frac{1}{\kappa}\left[P_{0}, \rho\right] N_{i}+\frac{1}{\kappa} \epsilon^{i j m}\left[P_{j}, \rho\right] M_{m},
$$

and the adjoint actions of $N_{i}$ and $P_{0}$ satisfy

$$
\operatorname{ad}_{\mathrm{ad} N_{i}\left(P_{0}\right)}(\cdot)=\operatorname{ad}_{N_{i}}\left(\operatorname{ad}_{P_{0}}\right)(\cdot)-\operatorname{ad}_{P_{0}}\left(\operatorname{ad}_{N_{i}}\right)(\cdot)
$$

thus ensuring that (23) satisfies a deformed notion of covariance. In this sense the non-trivial notion of covariance encoded in the Hopf algebraic structure of $\kappa$-deformations allows one to evade the no-go results of [19].

Besides investigating the actual physical viability of an evolution equation like (23) (checking, for example, whether it preserves positivity of the density matrix and further understanding the interplay between hermiticity and antipode from a quantum mechanical point of view), the most exciting development would be to phenomenologically constraint such models of deformed quantum evolution. As it stands, experimental searches for departures from ordinary time evolution in the form a Linbdlad equation have been ongoing for decades and have reached a striking sensitivity especially in precision measurements of neutral kaons and of the kaon-antikaon system, $K^{0}-\bar{K}^{0}$ [30]. It turns out that in order to properly appreciate the effects that a deformed evolution like (23) might have on the $K^{0}-\bar{K}^{0}$ system it will be crucial to have a clearer picture of the various ways the new features of deformed symmetries might alter the standard description of their states and evolution. In particular the role of discrete symmetries is central in this context and indeed the $K^{0}-\bar{K}^{0}$ system provides also a sensitive experimental probe for departures from CPT symmetry [30]. To this end in the final part of this contribution we will illustrate a first attempt at the formulation of discrete symmetry in a $\kappa$-deformed setting.

\section{Deformed discrete symmetries}

We report here on our recent proposal [31] to construct discrete symmetries associated to deformed symmetries based on group valued momenta and in particular for $\kappa$-Poincaré. The strategy followed in [31] has been to look for consistency with basic physical requirements that such discrete transformations should satisfy.

We start from parity and time-reversal transformations. The main physical requirement for the action of a parity transformation on momenta is that the total linear momentum of a one-particle state and its parity image must vanish. Following the discussion of Section 3 we see that such requirement leads to the parity image of the space translation generators to transform via their antipode

$$
\mathbb{P}: P_{i} \rightarrow S(P)_{i}
$$

In order to define the action of the parity operator $\mathbb{P}$ on the remaining symmetry generators we supplement the input from the physical requirement from (27) with two algebraic consistency conditions. The first is that antipode mapping of one generator requires antipode mapping of all the other symmetry generators in order to preserve the structure of the commutators of the algebra. The second is a "correspondence" requirement that in the limit $\kappa \rightarrow \infty$, in which one obtains the usual Poincaré algebra, the usual action of discrete transformations on symmetry generators should be recovered. These 
conditions determine the following parity transformations on the elements of the $\kappa$-Poncare algebra:

$$
\begin{array}{r}
\mathbb{P}\left(P_{i}\right)=S(P)_{i}=-P_{i}+\frac{P_{0} P_{i}}{\kappa}+O\left(\frac{1}{\kappa^{2}}\right) ; \quad \mathbb{P}\left(P_{0}\right)=-S(P)_{0}=P_{0}-\frac{\mathbf{P}^{2}}{\kappa}+O\left(\frac{1}{\kappa^{2}}\right) \\
\mathbb{P}\left(M_{i}\right)=-S(M)_{i}=M_{i} ; \quad \mathbb{P}\left(N_{i}\right)=S(N)_{i}=-N_{i}+\frac{1}{\kappa}\left(-P_{0} N_{i}+\epsilon_{i j k} P_{j} M_{k}\right)+O\left(\frac{1}{\kappa^{2}}\right) .
\end{array}
$$

Notice how the generator of rotations $M_{i}$ remains untouched by parity, as in the standard case, while the generators of boosts acquire energy-momentum dependent terms. One can proceed in a similar fashion for the time reversal transformation operator but now requiring that in the limit $\kappa \rightarrow \infty$ the sign of the generator of rotations $M_{i}$ should be changed. The resulting time reversal of symmetry generators is given by

$$
\begin{array}{rr}
\mathbb{T}\left(P_{i}\right)=S(P)_{i}, & \mathbb{T}\left(P_{0}\right)=-S(P)_{0} \\
\mathbb{T}\left(M_{i}\right)=S(M)_{i}, & \mathbb{T}\left(N_{i}\right)=-S(N)_{i}
\end{array}
$$

in which the only difference from (28) is the change in sign in the time reversed rotation and boost generators.

To complete the picture we should construct an appropriate charge conjugation operator. To this extent let us recall that for a complex quantum scalar field anti-particle states belong to the complex conjugate space $\overline{\mathcal{H}}$ associated to the usual one-particle Hilbert space $\mathcal{H}$ [32]. The ordinary charge conjugation operator $\mathbb{C}$ provides a map bewteen these two spaces

$$
\mathbb{C}: \phi(k) \in \mathcal{H} \rightarrow \bar{\phi}(-k) \in \overline{\mathcal{H}}
$$

where $\phi(k)$ and $\bar{\phi}(k)$ are representatives of particle and antiparticle states in terms functions of on-shell momenta. The crucial point to note is that the complex conjugate space $\overline{\mathcal{H}}$ is isomorphic to the dual Hilbert space $\mathcal{H}^{*}[31]$ and thus symmetry generators must act on antiparticle states via their antipodes. Imposing that in the $\kappa \rightarrow \infty$ one recovers the ordinary charge conjugation map we obtain

$$
\begin{gathered}
\mathbb{C}\left(P_{i}\right)=-S(P)_{i}, \quad \mathbb{C}\left(P_{0}\right)=-S(P)_{0} \\
\mathbb{C}\left(M_{i}\right)=-S(M)_{i}, \quad \mathbb{C}\left(N_{i}\right)=-S(N)_{i} .
\end{gathered}
$$

We thus see that all three discrete symmetries act on boosts and translations in a non-trivial way with the leading order departures from their usual actions suppressed by the inverse UV scale $\kappa$. Combining these relations together we can finally write down the explicit action of the deformed CPT operator on the generators of the $\kappa$-Poincaré algebra

$$
\begin{aligned}
& \mathbb{C P T}\left(P_{i}\right)=P_{i}-\frac{P_{0} P_{i}}{\kappa}+O\left(\frac{1}{\kappa^{2}}\right), \quad \mathbb{C P T}\left(P_{0}\right)=P_{0}-\frac{\mathbf{P}^{2}}{\kappa}+O\left(\frac{1}{\kappa^{2}}\right) \\
& \mathbb{C P T}\left(M_{i}\right)=-M_{i}, \quad \mathbb{C P T}\left(N_{i}\right)=-N_{i}+\frac{1}{\kappa}\left(-P_{0} N_{i}+3 P_{i}+\epsilon_{i j k} P_{j} M_{k}\right)+O\left(\frac{1}{\kappa^{2}}\right) .
\end{aligned}
$$

In summary we have showed that symmetry deformations involving a non-trivial antipode imply a deformed action of discrete symmetries and of the CPT operator on symmetry generators. Let us notice that in the framework we illustrated the same structures which determine departures from ordinary CPT symmetry are also responsible for a generalized quantum evolution allowing, in principle, a map of pure states into mixed states. We should point out that this scenario differs from models in which possible fundamental decoherence is linked to violations of CPT symmetry according to a known 
theorem by Wald $[33,34]$. In our framework CPT symmetry is not broken, we do have a well defined action of the CPT operator but this is deformed in the same way the action of translations and boosts on states and operators is deformed.

Of course, as for the generalized quantum evolution discussed in Section 5, much more needs to be understood for these new discrete symmetries starting from their action on fields of generic spin and the corresponding quantum states. Also in this case it will be very interesting to explore the possible phenomenological signatures of these departures from ordinary discrete transformations. The neutral kaon system, being already an extremely sensitive probe of for CPT violations, will be the main target of our future investigations.

\section{Discussion}

We illustrated how deformed symmetries associated to group valued momenta naturally predict departures from ordinary quantum evolution and CPT at energy scales of the order of the deformation parameter $\kappa$ determined by the curvature of the momentum group manifold. What we showed are just the first steps illustrating two novel theoretical developments in the field of symmetry deformations and much still remains to be done to fully appreciate the actual physics beyond deformed quantum evolution and discrete symmetries. Certainly the most remarkable aspect of this new window on fundamental decoherence and departures from CPT symmetry is the potential to phenomenologically constrain the model with precision measurements of neutral kaons. Over the past fifteen years virtually all of the efforts concerning the phenomenology of models based on departures from standard relativistic kinematics have focused on establishing bounds from the highest energy astrophysical observation (see e.g. [35, 36]). The theoretical developments reported here open the way to new potential experimental constraints for $\kappa$-deformed symmetries, one of the most studied models of deformed relativistic symmetries.

\section{References}

[1] S. W. Hawking, Commun. Math. Phys. 43, 199 (1975) [Erratum-ibid. 46, 206 (1976)].

[2] S. W. Hawking, Phys. Rev. D 14, 2460 (1976).

[3] S. W. Hawking, Commun. Math. Phys. 87, 395 (1982).

[4] J. R. Ellis, J. S. Hagelin, D. V. Nanopoulos and M. Srednicki, Nucl. Phys. B 241, 381 (1984).

[5] T. Banks, L. Susskind and M. E. Peskin, Nucl. Phys. B 244, 125 (1984).

[6] G. Lindblad, Commun. Math. Phys. 48, 119 (1976).

[7] J. R. Ellis, J. L. Lopez, N. E. Mavromatos and D. V. Nanopoulos, Phys. Rev. D 53, 3846 (1996) [hep-ph/9505340].

[8] A. Di Domenico, arXiv:1608.00241 [hep-ph].

[9] A. Staruszkiewicz, Acta Phys. Polon. 24, 735 (1963).

[10] S. Deser, R. Jackiw and G. 't Hooft, Annals Phys. 152, 220 (1984).

[11] H. J. Matschull and M. Welling, Class. Quant. Grav. 15, 2981 (1998) [arXiv:gr-qc/9708054].

[12] B. J. Schroers, PoS QG -PH, 035 (2007) [arXiv:0710.5844 [gr-qc]].

[13] M. Arzano, D. Latini and M. Lotito, SIGMA 10, 079 (2014) [arXiv:1403.3038 [gr-qc]].

[14] M. Arzano, Phys. Rev. D 77, 025013 (2008) [arXiv:0710.1083 [hep-th]].

[15] M. Arzano, arXiv:1212.1097 [hep-th].

[16] M. Arzano, Phys. Rev. D 90, no. 2, 024016 (2014) [arXiv:1403.6457 [hep-th]]. 
[17] M. Arzano, J. Kowalski-Glikman and T. Trzesniewski, Class. Quant. Grav. 31, no. 3, 035013 (2014) [arXiv:1305.6220 [hep-th]].

[18] H. Ruegg and V. N. Tolstoi, Lett. Math. Phys. 32, 85 (1994) [hep-th/9406146].

[19] M. Srednicki, Nucl. Phys. B 410, 143 (1993) [hep-th/9206056].

[20] J. Lukierski, A. Nowicki and H. Ruegg, Phys. Lett. B 293, 344 (1992).

[21] G. Amelino-Camelia, L. Smolin and A. Starodubtsev, Class. Quant. Grav. 21, 3095 (2004) [hepth/0306134].

[22] J. Kowalski-Glikman, hep-th/0312140.

[23] S. Majid and H. Ruegg, Phys. Lett. B 334, 348 (1994) [hep-th/9405107].

[24] J. Kowalski-Glikman and S. Nowak, hep-th/0411154.

[25] J. Kowalski-Glikman and S. Nowak, Class. Quant. Grav. 20, 4799 (2003) doi:10.1088/02649381/20/22/006 [hep-th/0304101].

[26] J. Lukierski, H. Ruegg and V. N. Tolstoi, IC-94-250, C94-02-14.1.

[27] J. Kowalski-Glikman and S. Nowak, Phys. Lett. B 539, 126 (2002) [hep-th/0203040].

[28] M. Arzano, Phys. Rev. D 83, 025025 (2011) [arXiv:1009.1097 [hep-th]].

[29] A. Borowiec and A. Pachol, J. Phys. A 43, 045203 (2010) [arXiv:0903.5251 [hep-th]].

[30] A. Di Domenico et al. [KLOE Collaboration], Found. Phys. 40, 852 (2010).

[31] M. Arzano and J. Kowalski-Glikman, Phys. Lett. B 760, 69 (2016) [arXiv:1605.01181 [hep-th]].

[32] Robert Geroch, Quantum Field Theory: 1971 Lecture Notes, Minkowski Institute Press, Montreal 2013

[33] N. E. Mavromatos, PoS KAON (2008) 041 [arXiv:0707.3422 [hep-ph]].

[34] R. M. Wald, Phys. Rev. D 21, 2742 (1980).

[35] G. Amelino-Camelia and T. Piran, Phys. Rev. D 64, 036005 (2001) [astro-ph/0008107].

[36] G. Amelino-Camelia, Living Rev. Rel. 16, 5 (2013) [arXiv:0806.0339 [gr-qc]]. 